\title{
Estudio de nuestro pasado prehistórico desde una perspectiva de género
}

A study of our prehistoric past from a gender perspective

O estudo do nosso passado pré-histórico a partir de uma perspectiva de género

\author{
Ivel Carolina Urbina-Medina \\ Antropóloga \\ Museo Antropológico "Francisco Tamayo Yépez" de Quíbor \\ Red de Conocimientos Antropológicos \\ Venezuela \\ Recibido: 22/10/2019 - Aceptado: 14/01/2020
}

Resumen

Este artículo propone analizar y reflexionar en torno al sesgo androcéntrico con el que se ha constituido las disciplinas históricas y en particular la arqueología, ciencia que estudia las sociedades extintas a través de sus huellas materiales, en el entendido de que las repercusiones que tienen el estudio y las interpretaciones de nuestro pasado americano ocasionan un entendimiento y razonamiento en nuestro presente. Se señala que la arqueología ha cumplido un papel de complicidad en la reproducción y naturalización de los estereotipos de género impuestos en nuestra sociedad, que terminan creando y legitimando relaciones de asimétricas entre individuos y colectivos. A partir de una investigación de tipo bibliográfico, enfocada desde una perspectiva de género y feminista, el propósi-

to de este artículo ha sido hacer un recorrido de las discusiones dentro de la arqueología sobre el género y cómo se tra-

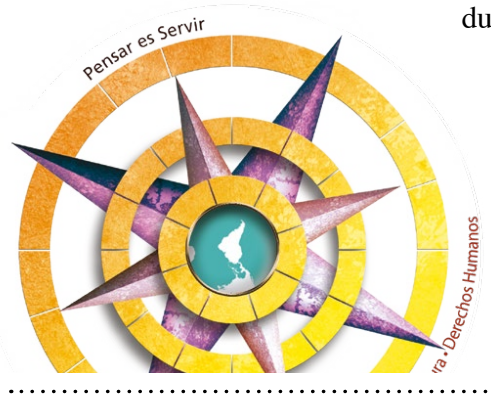
esto en la praxis arqueológica y, como consecuencia, en el entendimiento de nuestro pasado prehispánico. Ello, con la finalidad de aportar propuestas y cuestionamiento que sirvan para aprehender y entender nuestro pasado histórico desde una perspectiva decolonial, reconociendo que es 
parte de nuestra labor académica reivindicar a los colectivos históricamente excluidos en la producción intelectual, en este caso, los pueblos indígenas y las mujeres y, a su vez, brindarle el reconocimiento a los saberes ancestrales como alternativas para la construcción de una sociedad distinta.

Palabras claves: Arqueología, pueblos prehispánicos, perspectiva de género, arqueología, ciencias históricas, Latinoamérica.

\begin{abstract}
The purpose of the research presented is to analyze and reflect on the androcentric bias with which historical disciplines have been constituted and in particular archeology, a science that studies extinct societies through their material footprints; understanding that, the repercussions of the study and the interpretations of our American past cause an understanding and reasoning in our present. In this sense, archeology has played a role of complicity in the reproduction and naturalization of gender stereotypes imposed in our society, which end up creating and legitimizing asymmetric relationships between individuals and groups. It is based on a bibliographical research, focused from a gender and feminist perspective, whose purpose has been to review the discussions within archaeology about gender and how this translates into archaeological practice and, as a consequence, into the understanding of our pre-Hispanic past. The purpose is further contributing with proposals and questioning that serve to apprehend and understand our historical past from a decolonial perspective, understanding that it is part of our academic work to reclaim groups historically excluded in intellectual production, in this case indigenous peoples and women and, in turn, recognize ancestral knowledge as alternatives for the construction of a society different from the current one.
\end{abstract}

Keywords: Archeology, Prehispanic peoples, gender perspective, historical sciences, Latin America

\title{
Resumo
}

O objetivo da pesquisa apresentada é analisar e refletir sobre o viés androcêntrico com que as disciplinas históricas foram constituídas e em particular a arqueologia, uma ciência que estuda sociedades extintas através de suas pegadas materiais; entendendo que, as repercussões do estudo e as interpretações de nosso passado americano causam uma compreensão e um raciocínio em nosso presente. Neste sentido, a arqueologia tem desempenhado um papel de cumplicidade na reprodução e naturalização dos estereótipos de gênero impostos em nossa sociedade, que acabam por criar e legitimar relações assimétricas entre indivíduos e grupos. Ela se baseia em uma pesquisa bibliográfica, focalizada a partir de uma perspectiva de gênero e feminista, cujo objetivo tem sido rever as discussões dentro da arqueologia sobre gênero e como isto se traduz em prática arqueológica e, como consequiência, na compreensão de nosso passado

20 Estudio de nuestro pasado prehistórico desde una perspectiva de género Ivel Carolina Urbina-Medina

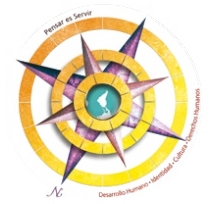


pré-hispânico. O objetivo é contribuir ainda mais com propostas e questionamentos que servem para apreender e compreender nosso passado histórico a partir de uma perspectiva descolonial, entendendo que faz parte de nosso trabalho acadêmico recuperar grupos historicamente excluídos na produção intelectual, neste caso povos indígenas e mulheres e, por sua vez, reconhecer os conhecimentos ancestrais como alternativas para a construção de uma sociedade diferente da atual.

Palavras chave: Arqueologia, povos pré-hispânicos, perspectiva de gênero, ciências históricas, América Latina

\section{Introducción}

Este artículo tiene como propósito hacer un recorrido y adentrarse en las problemáticas que se han venido desenvolviendo desde los años 1970 hasta el presente dentro de la arqueología como disciplina antropológica, entendida esta como una ciencia social que estudia e interpreta a las sociedades y sus culturas. Defino la arqueología como una disciplina científica que se encarga de estudiar las sociedades pretéritas a través de sus restos y huellas materiales. Dicha disciplina ha pasado por varias etapas distintas en cuanto a su desarrollo, cada cual resultante de la crítica y el cuestionamiento de su predecesora; para los efectos de esta investigación nos enfocaremos con lo que se conoce dentro de la arqueología como postprocesualismo, el cual se puede entender como distintas perspectivas y propuestas alternativas a la escuela arqueológica llamada nueva arqueología o procesualismo, en este sentido, no nos dedicaremos a dar cuenta de todas su multiplicidad, sino que nos detendremos predominantemente en una de ellas, la llamada arqueología del género y su importancia para el entendimiento de nuestro pasado prehispánico y nuestro presente. La arqueología del género, se encarga de lo siguiente:

Examinar las variaciones en los sistemas de las relaciones de género en la prehistoria, la forma en que se produjeron, las maneras en las que se reprodujeron y su papel en la dinámica social, investigar como intervienen la cultura material en la construcción de las relaciones de género e identificar como se inserta el género en los discursos materiales. (RodríguezShadow, 2007, citado por Castillo Bernal, 2011, p. 19)

El surgimiento de esta área de estudio se debe a los acontecimientos socio-políticos que se gestaron en Estados Unidos de América, a partir 
de 1970, con el movimiento feminista que ocasionó un fuerte cuestionamiento en el ámbito académico dentro de las ciencias sociales y su aporte en la legitimación y reproducción de las relaciones desiguales que se presentan en Occidente, entre hombre y mujeres en su diversidad de expresiones. Por lo tanto, la arqueología no se vio fuera de este razonamiento y empezó a reflexionar cómo había sido su labor hasta ese momento, cómo se estructuraba teóricamente y cómo afectaba a la sociedad actual.

Consecuente con todas estas discusiones, se funda esta línea de investigación propiamente dicha; sin embargo, sigue teniendo un desarrollo muy lento en comparación con otras ciencias, debido a que esta tiene dificultad, por un lado, para integrar teorías sociales a sus interpretaciones y, por otro, para poder aprehender el pasado en - términos metodológicos - en cuanto al género, debido a las cualidades de su objeto de estudio, por lo que continua en un proceso de construcción de las herramientas necesarias para esta labor. Ello implica una reestructuración de la arqueología como disciplina, lo cual sigue siendo una tarea sin terminar.

De igual forma, las organizaciones e individualidades arqueológicas que emprendieron esta labor, entendiendo su posición elitista y legitimadora como ciencia social en cuanto a la construcción y reproducción de significados e idiosincrasias en las sociedades del presente, deciden politizar su discurso y quehacer como una característica intrínseca a su labor.

El antropólogo Emanuel Amodio (1999) señala que la construcción del pasado y el presente siempre está en un continuo feedback representado por los intereses y el contexto en el que se encuentre el investigador o investigadora que esté realizando y escribiendo su interpretación. En este sentido, al escribir o narrar las relaciones de género en sociedades del pasado, en donde los grupos científicos representan tanto a las mujeres como a los hombres y usan como marco referencial los patrones y comportamientos de la sociedad occidental actual, reproducen y naturalizan los estereotipos de género, $\mathrm{y}$ terminan creando y legitimando relaciones asimétricas entre las personas del presente.

De igual forma, poder cuestionar el sesgo androcéntrico con el cual abordamos a nuestras generaciones antepasadas nos permite entender que las relaciones humanas $-\mathrm{y}$ las de género, en este caso - no fueron las mismas siempre, sino que cada

22 Estudio de nuestro pasado prehistórico desde una perspectiva de género Ivel Carolina Urbina-Medina

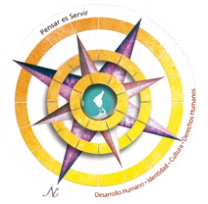


cultura las construye desde sus particularidades históricas y, por lo tanto, no son naturales ni inmutables. Todo esto nos permite difundir y educar a las nuevas generaciones para la construcción de una sociedad más equitativa y justa.

\section{Antecedentes y contexto histórico}

En primer lugar, comenzaremos hablando del contexto sociocultural que permitió el surgimiento de un análisis del género en todas las ciencias sociales. En este sentido, hay que ubicarse en los años 80 en Estados Unidos, en donde ya había transcurrido la primera oleada feminista, que tenía como principio la visibilización de las mujeres en todos los campos de la sociedad, el político, económico y académico; no obstante, no fue sino hasta esta década cuando empieza a emerger un cuestionamiento teórico a las estructuras de dominación que se imponían a las mujeres; el tiempo en el cual emerge el concepto de género. Este se define en los siguientes términos:

La propia descripción de un individuo y la adscripción que otros hacen de él o ella una o varias categorías de género específicas sobre la base de la diferencia sexual socialmente percibida ... por necesidad está culturalmente e históricamente determinadas.

(Díaz-Abreu, 2005 p. 14)

Empieza así una revolución en el plano académico dentro de las ciencias sociales, ya que surge un cuestionamiento de su quehacer.

Su crítica a los modelos hegemónicos de conocimiento occidental, incluyendo el científico, ha permitido develar la marcada visión androcéntrica y heterosexista, y en consecuencia occidental, eurocéntrica y elitista, que subyace acríticamente a los conceptos, procedimientos e interpretaciones de nuestra vida cotidiana y profesional. (Navarrete, 2008, p. 137)

En la antropología, igualmente, a partir de los movimientos feministas, se empezó a realizar una revisión de sus teorías y de su método de acercamiento al Otro u Otra, con respecto específicamente a las mujeres. En este sentido, hubo un pronunciamiento al sesgo masculino en la escritura etnográfica y la teoría, puesto que el androcentrismo deforma los resultados del trabajo de campo y provoca una distorsión de las interpretaciones, al equiparar la propia sociedad Occidental a cualquier otra sociedad a estudiar, sea del pasado o del presente, por lo que Henrietta Moore (2009, p. 16) expresa: "el androcentrismo no existe únicamente 
porque la mayoría de etnógrafos y de informantes sean varones, sino porque los antropólogos y antropólogas se basan en modales masculinos presentes en otras culturas". Asimismo, como paso lógico, el foco de atención pasó de ser la mujer al "género" como categoría de análisis, ya que "las diferencias biológicas entre el hombre y la mujer sólo tienen sentido dentro de sistemas de valores definidos culturalmente" (Henrietta Moore, 2009, p. 28).

Se volvió necesario estudiar a las mujeres dentro de estas relaciones de dominación discursivas, puesto que muy poco se había analizado y escrito sobre esto, para después pasar a estudiar las relaciones de género entre las distintas sociedades, dado que el amplio espectro de los roles de comportamientos entre individuos dentro de las sociedades demuestra su grandísimo grado de maleabilidad, sin importar lo que digan las predisposiciones biologicistas.

En este sentido, "Todas las sociedades conocidas reconocen y elaboran algunas diferencias entre los sexos ... en todas partes hay tareas, modales y responsabilidades características asociadas primordialmente a las mujeres o a los hombres" (Rosaldo, 1979, p. 154), aunque, no necesariamente binaria, puesto que las posibilidades son múltiples dependiendo de la cultura y cosmología de la sociedad en cuestión. En este sentido, en las sociedades occidentales, las mujeres tienden a ocupar una posición inferior con respecto a los hombres, puesto que existe una infravalorización del ser y la labor femeninas, lo que se veía reflejado, de manera inconsciente o no, en los trabajos antropológicos.

Esto es lo que Pierre de Bourdieu (2000) llamó como dominación masculina: estructuras, por lo tanto inconscientes, dentro de los seres sociales que continuamente se están legitimando a través de las mismas prácticas que esta determina, consideradas como universales y naturales, consecuencia del trabajo continuo de reproducción de modelos, tanto en los individuos, colectivos como en las instituciones; lo que el autor también acuñó como "violencia simbólica", es decir, "violencia amortiguada, insensible, e invisible para sus propias víctimas, que se ejerce esencialmente a través de los caminos puramente simbólicos de la comunicación y del conocimiento" (Bourdieu, 2000, p. 12). Propios de la producción académica y científica, tan eficaces que solo su concientización no basta para romperlos.

24 Estudio de nuestro pasado prehistórico desde una perspectiva de género Ivel Carolina Urbina-Medina

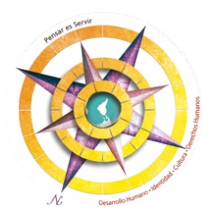


Esto no implica, primero, que las actividades femeninas no hayan sido primordiales para el desenvolvimiento normal de la sociedad, puesto que sus labores son igualmente necesarias. Y segundo, las relaciones entre ambos sexos no son iguales en todas las sociedades ni a través del tiempo, por lo que las mujeres han podido transitar por diversos ámbitos sociales en la historia humana.

Avanzando en nuestro razonamiento, el cuestionamiento se presentó en todas las ciencias sociales, no solo con respecto al género, sino en su quehacer mismo. En este sentido, en el plano de la arqueología, la cual estaba sumergida en un paradigma positivista, con aspiraciones cientificista que intentaba aprehender el pasado hipotético-deductivamente con la intención de crear leyes generales sobre el desarrollo social y cultural en el pasado humano y así, equipararse a las ciencias naturales.

Como consecuencia de todos estos conflictos, un grupo de arqueólogas empezaron, de igual forma, a criticar el sesgo androcéntrico con el cual se ha desenvuelto esta disciplina. El principal catalizador fue la obra de R.B Lee y I. DeVore titulada Hombre cazador en 1968, la cual ejemplificaba cómo se entendía el pasado hasta el momento. En resumidas cuentas: primero, se presumía de manera rígida la división sexual del trabajo, ya que existe la tendencia a asociar ciertos artefactos a ciertas actividades, bien sean masculinas o femeninas, produciendo inferencias simplistas acerca de la vida social de los grupos prehispánicos. Segundo, se le asignaban diferentes valores sociales a cada actividad, especulando que las actividades masculinas son más importantes para el desarrollo de la sociedad en contraposición de las femeninas.

Y por último, les atribuyen vinculaciones tanto biológicas como psicológicas para reafirmar sus conclusiones, véase, los hombres tienen mejores condiciones físicas para realizar labores como la caza y las mujeres psicológicamente son más "sensibles" para desarrollar este tipo de actividad (Conkey y Spector, 1984). De ahí que, junto a los movimientos feministas ya mencionados, se hizo necesaria una arqueología que visualizara los conflictos teóricos y metodológicos de la concepción de "mujer" en el estudio del pasado.

\section{El género en el estudio del pasado}

Cuando se estudian sociedades pretéritas, se debe tener cuidado de las 
perspectivas teóricas que se emplean, debido a que en su mayoría no están pensadas para utilizarse en el pasado. Este ha sido un gran debate en las ciencias históricas y, particularmente, en los estudios sobre género. Por este motivo, la historiadora Joan Scott (1990) critica los estudios de género en la historia, puesto que se han limitado a las descripciones de las mujeres en el pasado, sin un sustento teórico.

Asimismo, observa que cuando se escribe sobre el género se utiliza para referirse a las relaciones entre los sexos, y no se transversaliza a otros ámbitos sociales como el político, económico, religioso, etc., es decir, no es utilizado para explicar o entender mejor estas relaciones sociales, sino que se limita describir estas dinámicas, específicamente las desigualdades entre hombres y mujeres.

Por esto, Scott (1990) propone que las relaciones de género deben ser entendidas como elementos constitutivos de las relaciones de poder de cualquier ámbito, y este último debe ser comprendido dentro de una multiplicidad de variantes en las cuales se desenvuelve. En otras palabras, consiste en despegarnos de la concepción tradicional de poder: unificado, centralizado y desde arriba, sino que se dan distintas formas de trasgresión y resistencia. En consecuencia, las relaciones de género son primordiales para el entendimiento cabal de las estructuras sociales e identitarias de una sociedad determinada.

De acuerdo con la definición de género de Scott (1990), para aplicar el concepto de género, en los estudios históricos, hay que considerar que está dividido en cuatro elementos, los cuales están interrelacionados entre sí de manera diversa dependiendo del contexto y del momento histórico en donde se sitúe. Estos elementos son: está cargado de símbolos socialmente constituidos y su capacidad de evocar diversidad de representaciones. Segundo, está legitimado a través de conceptos normativos que regulan las interpretaciones que se le dan, las cuales pueden ser variadas y contradictorias. Tercero, debe situarse dentro de nociones políticas, institucionales y organizacionales en donde se producen y se reproducen constantemente. Y finalmente, es constituyente de las identidades subjetivas individuales y grupales, históricamente construidas (Scott, 1991, pp. 45-46).

Por lo tanto, "el género ya no puede ser ignorado y debe ser tenido en cuenta como una categoría analítica que, junto a la raza, etnia, clase o casta, es crucial en la construcción

26 Estudio de nuestro pasado prehistórico desde una perspectiva de género Ivel Carolina Urbina-Medina

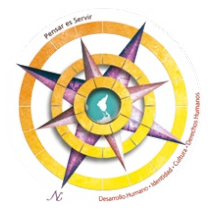


de identidad de grupo y de las estructuras de poder" (Lewellen, 2003, p. 185), debido a que el amplio espectro de los roles de comportamientos entre individuos dentro de las sociedades, demuestra el grandísimo grado de maleabilidad, sin importar lo que se perciba en las predisposiciones biológicas.

\section{La arqueología del género}

En la arqueología del género, su principal interés es la crítica a los parámetros teóricos y metodológicos que estructuran esta disciplina hasta ese momento, por lo que es interesante visualizar cuáles fueron las principales discrepancias y críticas que le hacen las académicas feministas.

En primer lugar, está el período de la toma de conciencia de los sesgos ideológicos y androcéntricos de las interpretaciones del pasado. Entiéndase, el lenguaje e imágenes masculinas para referirse al pasado, ya sea utilizar el término "hombre" para referirse las sociedades humanas en general; asimismo, para referirse a las actividades "masculinas" siempre se expresa de manera activas y positivas para el desarrollo de la sociedad y las actividades "femeninas" como pasivas y de poca importancia. De igual manera, se observa en las categorías utilizadas para referirse a roles de poder como, por ejemplo, caciques (hombre líder), pero sin considerar la posibilidad de que las mujeres pudieron haber sostenido esta posición (Díaz-Andreu, 2005). Posteriormente, se presentó la necesidad de criticar y denunciar políticamente la arqueología como comunidad científica y sus implicaciones en la construcción de identidades y la legitimación de las relaciones de dominación-subordinación que se reproducen en el presente.

Consecuentemente, el siguiente paso es formular una alternativa teórica y metodológica para estudiar el pasado desde una perspectiva de género, ya que permite una interpretación menos parcial de las realidades posibles y la disminución del sesgo moderno y occidental de las conclusiones a las que llegan los distintos grupos de especialistas. En este sentido, se evocan dos tópicos: el primero, demostrar que las diferencias y desigualdades de género que percibimos en occidente no son universales ni en el presente y mucho menos en el pasado. Y segundo, al dar cuenta de las relaciones de dominación en algunas sociedades pretéritas, se pueda trazar su trayectoria hasta el presente y así crear políticas de cambio (Navarrete, 2008). 
Por último, se puede hablar de una etapa de crítica y reflexión, tanto a lo externo como a lo interno de la disciplina, en la cual hacen apariciones las perspectivas planteadas por personajes como Judith Butler (2007), quien asimismo señala que las feministas han asumido la existencia de un estereotipo de "mujer" para los intereses y objetivos políticos propios. Por lo que surge un cuestionamiento del propio quehacer de la arqueología feminista, debido a que al intentar visibilizar a la mujer o sobrestimarla con intereses políticos se puede terminar por falsear las interpretaciones, lo que también significaría una reincidencia a la propia crítica que hacen estas a la arqueología tradicional.

Por lo tanto, el papel del género como categoría de análisis se hace tan imprescindible y útil; en palabras de Moore (2009, p. 20) "El concepto 'mujer' no puede constituir una categoría analítica de investigación antropológica $y$, por consiguiente, no pueden existir connotaciones analíticas en expresiones tales como 'situación de la mujer', 'subordinación de la mujer' o 'hegemonía del hombre' cuando se aplican universalmente"; aunque se refiera a los estudios de la antropología social y cultural, cabe perfectamente para el estudio de las sociedades del pasado.
No obstante, la arqueología del género ha tenido sus dificultades, y tal vez es lo que ha provocado un desarrollo más atrasado en comparación con otras ciencias sociales, y es principalmente su dificultad para visualizar a las mujeres en el pasado, debido a que las ciencias que interpretan el pasado no poseen los sujetos vivos para su estudio, sino que se valen de las huellas dejadas por estos para inferir en sus modos de vida. Por lo que no se trata solo de relacionar ciertos objetos con un sexo o el otro, sino que se vuelve necesario elaborar nuevas maneras de contemplar los datos arqueológicos.

En este sentido, en la obra Archaeology and the study of gender (1984), Conkey, junto a Spector, cuestionan el papel de la arqueología en la sociedad occidental: demuestran cómo crean -intencionalmente o nouna mitología en cuanto al pasado y, por consiguiente, una mitología del género con graves implicaciones políticas y educativas, lo que se traduce, como la justificación de creencias culturales sobre el significado de lo masculino y lo femenino, acerca de las capacidades de los hombres frente a las de las mujeres, las relaciones de poder entre sí y sobre sus papeles e importancias en la sociedad.

28 Estudio de nuestro pasado prehistórico desde una perspectiva de género Ivel Carolina Urbina-Medina 
Estas repercusiones se deben a la falta de marcos conceptuales y metodológicos para abordar el género, por lo que utilizan sus experiencias contemporáneas para abordar el pasado, "los arqueólogos conscientemente o no, son propagadores de una cultura y de ideas acerca del género particulares [las Occidentales] en sus interpretaciones y reconstrucciones del pasado" (Conkey y Spector, 1984, p. 2).

Esta es una crítica contundente a la arqueología y la antropología en general, debido a que atenta con la propia labor de esta ciencia, ya que se supone que estudia las diversidades culturales de las distintas sociedades $\mathrm{y}$, por consiguiente, no se debe posicionar en favor de ninguna ni criticar otras.

Si nuestras descripciones e interpretaciones de la vida de otras culturas simplemente reiteran nuestros propios supuestos acerca del género, minimizamos los esfuerzos hacia la explicación de la diversidad cultural, mientras que al mismo tiempo justificamos nuestras propias ideologías del género. (Conkey y Spector, 1984, p. 3)

Por otro lado, el género no se "encuentra" como si fuese un objeto, debido a que es una construcción y, como categoría analítica no es estática, "el análisis del mismo es un procesos dinámico y se refiere a un conjunto de funciones, prácticas y creencias cambiantes" (Conkey, 2001 , p. 343), por lo que es imprescindible hacer buenas preguntas, ya que existe lo que ella denominó como "método idolatría", una insistencia de atribuirle género o sexo a todo lo que se presente, por lo que hay que aprender a discernir a cuáles datos arqueológicos se les puede hacer análisis de género.

Otro cuestionamiento que se suscita, es cuando en la narrativa arqueológica no se relativiza ni se relaciona la categoría de género con otros conceptos como etnia, linaje, clase, etc., es decir, integrar la perspectiva interseccional a sus interpretaciones. Por ejemplo, la caracterización de lo femenino y sus labores no son las mismas cuando se es infante a cuando se es adulta o cuando se pertenece a distintos estamentos, rangos o clases.

De igual forma, en los contextos funerarios se visualizan estas mismas especulaciones, por ejemplo, cuando se consiguen fragmentos de vasijas en un enterramiento femenino, se infiere que fueron para que ellas preparan alimentos, pero cuando se hallan con un cadáver masculino, se piensa que fueron sus fabricantes o son ofrendas por su posición social. Por otro lado, cuando se consiguen 
armas se aluden inmediatamente como masculinas o las joyas como femeninas, pero cuando se consiguen en enterramientos masculinos se piensan que estos fueron comerciantes o hasta se consideran pruebas de la homosexualidad del sujeto (Díaz-Andreu, 2005, p. 33).

En resumidas cuentas, se puede decir que la narrativa arqueológica tradicional le presta mayor atención e importancia al hombre y a sus presuntas actividades que a las mujeres y a las labores "femeninas". En segundo lugar, a las mujeres se les relega, principalmente, a las actividades domésticas; mientras que a los hombres se les atribuyen labores de poder, lo que se traduce como una rígida conceptualización de la división sexual del trabajo. Dichos presupuestos son tomados de rasgos pertenecientes a su propia cultura, reflejados en la sociedad del pasado y supuestos como únicos $\mathrm{y}$ universales, lo que ha promovido la distorsión de la idea sobre las mujeres tanto como sobre los hombres (Spector, 1993).

\section{La arqueología del género para la decolonización de Nuestra América}

En Latinoamérica se ha vuelto necesario el estudio tanto del presente como del pasado, desde una perspectiva propia, construida por nuestros propios equipos de investigación científica social de esta región. Es necesaria la construcción de un discurso sobre nuestro pasado que reivindique nuestro desarrollo y que denuncie los atropellos y despojos históricamente sufridos por nuestros pueblos, desde la conquista hasta nuestros días; esto es fundamental para crear una conciencia y una existencia de nuestro ser y habitar decolonizado de las culturas foráneas impuestas.

De ahí que la arqueología, al estudiar sociedades que no dejaron un registro escrito para su acceso, de manera que su pasado solo puede ser aprehendido a través de sus huellas materiales y osteológicas, debe ser capaz de interpretar miles de años de historia humana mil veces mayor de lo que reseñan los textos desde la conquista. Se pueden llegar a conocer culturas y cosmologías no occidentales, que puedan servir de sustento para la elaboración de alternativas económicas, políticas y sociales para nuestra región; así como aditamento para la construcción de identidades propias ancladas a nuestro pasado indígena, como pueblos originarios de estas tierras.

Este pasado histórico debe ser tomado en cuenta por las ciencias

30 Estudio de nuestro pasado prehistórico desde una perspectiva de género Ivel Carolina Urbina-Medina

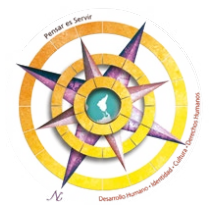


sociales, puesto que es un tiempo invisibilizado parcialmente por la epistemología moderna, la cual se ha encargado de fragmentar o ignorar nuestro pasado. La arqueología como disciplina puede decolonizar esta desposesión del tiempo. Morar en la periferia es morar en el pasado. En este sentido, Shepherd, Gnecco y Haber plantean el siguiente ejemplo:

El arqueólogo y el indígena entran en la misma sala, se sientan en la misma mesa, pero se entiende que ocupan el tiempo de otra manera: la ciencia, la modernidad, el desarrollo y la orientación hacia el futuro están con el arqueólogo mientras que el indígena habla por la cultura, la tradición y una preocupación performativa con identidades enraizadas en pasados imaginados. (2006, p. 3)

Por esta razón, como primer paso para crear una sociedad realmente libre de ataduras es menester la reivindicación de las personas o grupos históricamente invisibilizados y desplazados, y más nuestros pueblos indígenas sobrevivientes como personajes activos en nuestro presente. Este es un papel importante y una deuda pendiente desde las ciencias sociales y de la arqueología en este caso.

Por consiguiente, las mujeres y los sexo-diversos forman parte de estos colectivos y nuestra labor, desde las disciplinas que se encargan de estudiar el pasado, es publicar su importancia como integrantes de nuestras sociedades y, por lo tanto, revalorizar su intervención en el desarrollo y la reproducción social de nuestra historia y de lo que constituimos hoy en día, y lo que queremos convertirnos en el futuro.

Como se ha reseñado en todo este escrito, se vuelve no solo importante sino imprescindible el estudio del pasado desde una perspectiva de género anclada en las necesidades contextuales del presente. Debemos entender que el género no solo abarca lo femenino y lo masculino como únicas variables inmutables, sino que existe una multiplicidad de realidades individuales y colectivas que conviven y que, seguramente, fueron tan diversas en el pasado como en el presente, por lo que su visibilización es necesaria, si queremos crear una sociedad más equitativa y justa para la totalidad de sus habitantes.

\section{Consideraciones finales}

Como se ha observado en el presente trabajo, las ciencias históricas tradicionales ya no dan respuesta a todas las incógnitas y necesidades que han venido surgiendo en las últimas décadas dentro de estas disciplinas y en 
la sociedad en general, pues ninguna ciencia está al margen del contexto sociocultural en el que se desenvuelve, y mucho menos las ciencias sociales, por lo que fue necesario el surgimiento de una crítica de sus labores dentro de la reproducción y la legitimación de las relaciones sociales en el mundo occidental.

Por tanto, se ha integrado la toma de consciencia de la arqueología como parte de una construcción social al igual que sus interpretaciones, lo que implica la participación activa del investigador o investigadora, es decir, no existe una objetividad total y cientificista como se pretendía (y algunos grupos científicos todavía pretenden). En este sentido, asumen una posición política abiertamente, ya que saben cuáles son las repercusiones de sus interpretaciones en la sociedad actual.

Dicho lo anterior, la arqueología del género, la cual surgió gracias a los acontecimientos que se estaban suscitando por movimiento feminista, permitió: primero, que se reflexionara en torno el quehacer de las ciencias sociales, que muy poco se había hecho. Posteriormente, se comenzó un cuestionamiento de las estructuras de dominación que se rigen sobre las mujeres, y es cuando hace aparición del concepto de género, lo que significó un cambio cualitativo dentro de todas las ciencias sociales, así como en las disciplinas que estudian el pasado, lo que se tradujo en la organización de encuentros, se incentivó el debate y, en consecuencia, la necesidad de crear un cuerpo teórico y metodológico que sirviera para la interpretación del género en el pasado.

Para los equipos científicos nuestroamericanos, y quienes somos parte y apuntamos a la decolonización y a la reivindicación de nuestras sociedades, no se debe ignorar los siglos de opresión foránea, ni el pasado prehispánico de la región, puesto que en ese pasado se construyeron formas de vivir que han perdurado hasta el presente y las cuales son un ejemplo de la existencia de alternativas no occidentales ni capitalistas de ser, que deben servir de aditamento para la construcción de una sociedad distinta a la actual.

En conclusión, el pasado es construido con base en nuestra forma de ver el presente y en el contexto que corresponda a cada momento histórico, por lo que la arqueología también ha respondido a los intereses, normalmente de una élite determinada, que bajo su consigna de "ciencia" y "objetividad", ha calado en el sentido común de los individuos

32 Estudio de nuestro pasado prehistórico desde una perspectiva de género Ivel Carolina Urbina-Medina

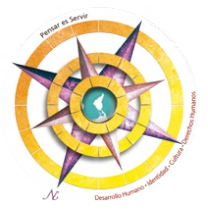


legitimando comportamientos de desigualdad. En otras palabras, no se trata solo de una lealtad política, sino de una perspectiva que lleva a ver el género como una mejor manera de conceptualizar la política.

\section{Referencias}

Amodio, E. (1999). Aproximaciones a un lugar de encuentro entre historia y antropología. En Emanuele Amodio (Ed.), La vida cotidiana en Venezuela durante el siglo XVIII (3-14). Dirección de Cultura LUZ.

Bourdieu, P. (2000). Dominación masculina. Editorial Anagrama.

Butler, J. (2007). El género en disputa: El feminismo y la subversión de la identidad. Paidós Ibérica.

Castillo Bernal, S. (2011). No a las etiquetas teóricas. La inserción de los estudios de género en la práctica arqueológica. En Miriam López Hernández y María Rodríguez Shadow (Editas.), Género y sexualidad en el México Antiguo (15-34). Centro de estudio de la Mujer. http://www.academia.edu/8714589/No_a_las_etiquetas_te\%C3\%B3ricas_.La_inserci\%C3\%B3n_de_los_estudios_de_g\%C3\%A9nero_en_la_pr\%C3\%A1ctica_ arqueol\%C3\%B3gica

Conkey, M. (2001). Thinking about with theory and method. En Cecilia Klein (Edit.), Gender in pre-hispanic American (341-362). Dumbarton Oaks.

Conkey, M. y Spector, J. (1984). Archaeology and the Study of Gender. En Michael Schiffer (Edit), Advances in Archaeological Method and Theory (1-38). Academic Press.

Díaz-Andreu, M. (2005). Género y arqueología: una nueva síntesis. En M. Sánchez Romero (Edit.), Arqueología y género (13-51). Editorial Universidad de Granada.

Lewellen, T. (2003). Introducción a la antropología política ( $3^{\mathrm{a}}$ ed.). Ediciones Ballaterra.

Moore, H. (2009). Antropología y feminismo. (5 ed.). Ediciones Cátedra.

Navarrete, R. (2008). Cucharas y picos: Contribución de la arqueología feminista al estudio del género. Revista venezolana de estudio de la mujer, 13(30), 133- 154.

Rosaldo, M. Z. (1979). Mujer, cultura y sociedad: Una visión teórica. En O. Harris y K. Young (Comps.), Antropología y feminismo (153-180). Editorial Anagrama.

Scott, J. (1990). El género: Una categoría útil para el análisis histórico. En Nash y Amelang (Editas.), Historia y género: Las mujeres en la Europa moderna y contemporánea. (2356). Alfons el magnanim.

Shepherd, N., Gnecco, C. y Haber, A. (2016). Arqueología y decolonialidad. Editores de Signo.

Spector, J. (1993). What this awl means. Feminist Archaeology at Wahpeton Dakota Villages. Minnesota Historical Society Press. 
